\title{
RELEVÂNCIA CLÍNICA DE BAIXOS TÍTULOS DE ALOANTICORPOS PREFORMADOS DETECTADOS EM PROVA CRUZADA SOMENTE POR CITOMETRIA DE FLUXO NO PRÉ-TRANSPLANTE RENAL
}

\author{
Clinical relevance of low levels of preformed alloantibodies detected by flow-crossmatch in \\ deceased kidney transplant
}

Michelon $T^{\prime}$, Schroeder $R^{2}$, Fagundes $I^{3}$, Canabarro $R^{3}$, Sporleder $H^{3}$, Rodrigues $H^{2}$, Silveira $J^{2}$, Montagner $J^{2}$, Garcia $V^{4}$, Keitel $E^{5}$, Neumann $J^{6}$, Graudenz $M^{7}$.

\begin{abstract}
RESUMO
O bjetivo: Avaliar a importância da prova cruzada pré-transplante positiva por citometria de fluxo e identificar subgrupos com potencial benefício para o primeiro ano pós-transplante renal. Pacientes e Métodos: Foram estudados 157 transplantes renais realizados após prova cruzada negativa por citotoxicidade contra linfócitos T e B. 0 soro pré-transplante foi retestado por citometria. Os desfechos clínicos foram: não-função primária ( $n=14 ; 8,9 \%)$, função retardada ( $n=107 ; 68,1 \%)$, rejeição aguda $(n=68 ; 43,3 \%)$ e perda do enxerto $(n=25$; $15,9 \%$ ), óbito $(n=16 ; 10,2 \%)$ e sobrevida do enxerto. A função do enxerto foi avaliada pela creatinina. Foram estudados subgrupos: présensibilizados $(n=24)$; diabetes $(n=29)$, rim +pâncreas $(n=22)$, doador limítrofe $(n=55)$, hipersensi bilizados $(n=8)$ e re-transplantes $(n=8)$. Foram empregados testes do Q ui Quadrado ou exato de Fisher, t Student e K aplan M eier. Para análises bivariadas considerou-se P $<0,05$ e para interações (Cochran-M antel-H aentzel) $P<0,10$. Resultados: H ouve 30\% ( $n=47)$ de citotoxicidade negativa contra linfócitos T e 36\% $(n=56)$ contra $B$ que eram positivas na citometria. A s características gerais e eventos clínicos pós-transplante foram semel hantes entre os pacientes citometria negativa e positiva. Pacientes pré-sensibilizados com citometria positiva tiveram creatinina superior $(2,0+0,7 \times 1,3+0,3$ $\mathrm{mg} / \mathrm{dl} ; \mathrm{P}=0,020)$ e menor sobrevida do enxerto $(80 \% \times 43 \% ; \mathrm{P}=0,074)$ em um ano comparados aos pré-sensibilizados com citometria negativa. A interação pré-sensibilização e citometria positiva foi significativa para perda do enxerto $(0 R=9,1 ; P=0,098)$ e óbito $(0 R=12,1$; $P=0,088)$. Os demais grupos não tiveram benefício na investigação por citometria. Conclusão: Pré-sensibilizados com prova cruzada positiva por citometria têm creatinina mais elevada e maior risco para perda do enxerto e óbito no primeiro ano pós-transplante renal.
\end{abstract}

Descritores: aloanticorpos, transplante renal, citometria de fluxo, prova cruzada

Grau acadêmico:

1. Médica nefrologista do Laboratório de Imunologia de Transplantes da Santa Casa de Porto Alegre, doutora em Patologia, Pesquisadora PRODOC-CAPES do Programa de PósGraduação em Patologia da Fundação Faculdade Federal de Ciências Médicas de Porto Alegre.

2. Bióloga do Laboratório de Imunologia de Transplantes da Santa Casa de Porto Alegre, Fundação Faculdade Federal de Ciências Médicas de Porto Alegre.

3. Farmacêutica do Laboratório de Imunologia de Transplantes da Santa Casa de Porto Alegre.

4. Médico nefrologista, Doutor em Nefrologia, diretor do serviço de Transplante Renal da Santa Casa de Porto Alegre.

5. Médica nefrologista do serviço de Transplante Renal da Santa Casa de Porto Alegre, Doutora em Clínica Médica.

6. Médico imunologista, Diretor do Laboratório de Imunologia de Transplantes da Santa Casa de Porto Alegre.

7. Médica patologista, Doutora em Patologia, Fundação Faculdade Federal de Ciências Médicas de Porto Alegre.

Instituição:

Fundação Faculdade Federal de Ciências Médicas de Porto Alegre

Santa Casa de Porto Alegre - Laboratório de Imunologia de Transplantes e Serviço de Transplante Renal

\section{Correspondência:}

Tatiana Michelon

Hospital Dom Vicente Scherer

Av. Independência, 75 - CEP 94035-075 - Porto Alegre/RS - Brasil

Tel/Fax: 55513214 8670/3214 8629

E-mail: tatimich@yahoo.com

Recebido em: 12/09/2005

Aceito em: 05/10/2005

\section{INTRODUÇÃO}

Desde 1964, a prova cruzada por citotoxicidade dependente de complemento $(C D C)$ tem sido a técnica padrão para a avaliação da presença de anticorpos anti-HLA específicos contra o doador antes de um transplante. A loanticorpos pré-formados assim detectados podem causar rejeição hiperaguda e perda imediata do enxerto. A nticorpos anti-HLA detectados após a exposição ao enxerto também têm potencial deletério e têm sido associados à rejeição aguda precoce, rejeição crônica e menor sobrevida do enxerto renal, cardíaco, hepático e corneano.

É razoável, portanto, se considerarmos que alguns eventos desfavoráveis ainda hoje observados no pós-transplante precoce, tais como não-função primária do enxerto (ENF) efunção retardada do enxerto ( $F R E$ ), possam resultar de algum grau de envolvimento humoral não diagnosticado através das técnicas tradicional mente empregadas na avaliação imunológica pré e pós-transplante.

0 emprego de técnicas mais sensíveis poderia permitir a detecção de anticorpos específicos contra o doador e potencialmente prejudiciais ao enxerto em títulos muito baixos, de outra forma não identificados. A técnica mais sensível para a detecção de aloanticorpos é a citometria de fluxo (CF), a qual vem sendo empregada na prática dos transplantes desde 1983. Esta tem sido a técnica-padrão para o diagnóstico de atividade humoral através da detecção de baixos 
títulos de aloanticorpos circulantes. Prova cruzada através desta técnica é capaz de detectar anticorpos anti-H LA em pacientes que tiveram resultado negativo em prova cruzada por CDC. Pode ser 50 vezes mais sensível do que a CDC e até 15 vezes mais sensível do que a CDC com anti-globulina humana ( $A G H)$.

No B rasil, nenhum centro de transplante tem rotineiramente tomado suas decisões clínicas baseado nos resultados de provas cruzadas por CF (PCCF). A pós mais de dez anos de experiência com a CDC em nosso centro, um grupo de pacientes submetidos a transplante renal com doador falecido com provas cruzadas negativas contra linfócitos T e B por CDC foram acompanhados, a fim de se estudar a relevância clínica de baixos títulos de aloanticorpos específicos contra 0 doador, detectados somente por PCCF imediatamente antes do transplante, e identificar eventuais subgrupos específicos de pacientes que se beneficiariam de avaliação rotineira por PCCF no pré-transplante.

\section{PACIENTES E MÉTODOS Pacientes}

Entre os 182 pacientes submetidos a transplante renal com doador falecido na Santa Casa de Porto A legre entre novembro de 2001 e outubro de 2003, 157 foram elegíveis para o presente estudo. Pacientes menores de 18 anos de idade $(n=13)$, ausência de células congeladas do doador $(n=8)$ ou de soro pré-transplante do receptor $(n=1)$, pacientes submetidos a transplante com prova cruzada positiva contra linfócitos $\mathrm{T}$ com antiglobulina humana (AGH) e ditiotreitol (DTT; T-CDC +A HG +DTT) $(n=1)$ ou contra linfócitos B com DTT ( $B-C D C+D T T, n=2$ ) no soro atual foram excluídos da análise.

$A$ avaliação imunológica pré-transplante incluiu a tipagem $H L A$, o teste de reatividade contra painel de células (PRA) e a prova cruzada pré-transplante por CDC. 0 teste de PRA inicialmente era realizado através de técnica de imunoensaio enzimático (ELISA, screening LAT-M ; O ne Lamda) e, quando este era positivo, realizava-se também o PRA quantitativo por CF. Todos os pacientes foram submetidos ao transplante após 0 resultado negativo de ambas as provas cruzadas por $C D C$ : T-CDC +AGH +DTT e B-CDC+DTT, simultaneamente. A avaliação do risco imunológico no momento do transplante também considerou a história de gestações, transfusões sanguíneas e transplantes prévios. A escolha do esquema de drogas imunossupressoras considerou a presença das características descritas acima mais o tipo de transplante (rim isolado ou transplante combinado) e tipo de doador falecido (tradicional ou limítrofe para a função do enxerto).

O soro estocado e previamente utilizado para a prova cruzada pré-transplante por CDC foi submetido à análise por CF retrospectivamente, após aprovação do estudo pelo Comitê deÉtica em Pesquisa da instituição. A s medidas de creatinina sérica foram realizadas conforme o protocolo institucional e as biópsias foram classificadas conforme os critérios de B anff 97.

\section{Prova Cruzada por Citometria de Fluxo}

Para a realização das provas cruzadas contra linfócitos T e B por CF aplicou-se o protocolo anteriormente publicado, em um equipamento FACSC alibur (B ecton Dickinson, San J ose, CA), utilizando o software CellQuest (Becton Dickinson, San J ose, CA) para a análise. A prova cruzada quantitativa foi realizada utilizando-se técnica com três cores, incluindo um conjugado anti-IgG humana marcado com ficoeritrina
(PE; Sigma, Saint Louis, M O) para identificar anticorpos IgG antidoador e anticorpos monoclonais marcados com isotiocianato de fluoresceína (FITC) dirigidos contra linfócitos T (anti-CD3; B ectonDickinson, San J ose, CA) eB (anti-CD20; B ecton-D ickinson, SanJ ose, CA ). Todas as reações foram comparadas a um controle positivo ea um controlenegativo. A média daintensidade de fluorescência $>40$ canais acima da média da intensidade de fluorescência do controle negativo foi considerada prova T positiva (mean channel shift $>40$ ). Testes contra linfócitos B foram classificados conforme o resultado da prova contra linfócitos T: a) mean channel shift $>60$ quando a prova T era negativa, e B ) mean channel shift >100 quando a prova T era positiva.

Para as análises comparativas, os pacientes foram divididos conforme os resultados da prova cruzada pré-transplante em PCCF- e PCCF +. Um paciente realizou PCCF apenas contra linfócitos $T$ devido à ausência de linfócitos totais congelados para a análise retrospectiva.

\section{Desfechos Clínicos}

Os eventos clínicos analisados incluíram: não-função primária do enxerto (ENF), função retardada do enxerto (FRE), rejeição aguda cel ular (RAC), creatinina sérica, óbito e perda do enxerto no primeiro ano pós-transplante. A função do enxerto foi avaliada pelos níveis séricos de creatinina coletados semanalmente no primeiro mês, quinzenalmente no segundo e terceiro meses e também no sexto e décimo segundo meses. Pacientes que apresentaram EN F foram excluídos da análise da função renal. E NF foi definido como os casos em que o enxerto nunca assumiu função suficiente para 0 abandono da diálise. FRE foi definida como a necessidade de diálise na primei ra semana pós-transplante. A sobrevida do enxerto foi avaliada mensalmente durante o primeiro ano pós-transplante e analisada de forma atuarial.

\section{Análise Estatística}

Todas as análises foram realizadas utilizando-se o software Statistical Package for Social Sciences (SPSS, versão 11.0). Os resultados foram descritos como porcentagem e média e desviopadrão. A significância estatística foi avaliada através de teste do Qui-quadrado ou exato de Fisher e t de Student. A sobrevida atuarial do enxerto foi estimada pelo método de K aplan M eier. A s análises de interação foram realizadas através do procedimento de Cockran-M antel-Haentzel. Consideram-se significativos valores de $\alpha=0,05$ para as comparações bivariadas e de $\alpha=0,10$ para as análises de interação.

\section{RESULTADOS \\ Estudo da População Geral}

Prova Cruzada por Citometria de Fluxo N egativa x Positiva:

A presença de anticorpos não diagnosticados por prova cruzada TCDC +A GH +DTT e/ou B - CD C +DTT foi observada em 75 pacientes. Isto representou uma incidência de $47,8 \%$ de pacientes com anticorpos em baixos títulos não detectados pela técnica tradicionalmente empregada imediatamente antes do transplante: $12 \%(n=19)$ contra linfócitos T, $18 \%(n=28)$ contra linfócitos B e $18 \%(n=28)$ contra linfócitos T e B simultaneamente. Desta forma, 30\% $(n=47)$ dos pacientes foram submetidos a transplante renal com anticorpos dirigidos contra linfócitos T e $36 \%(n=56)$ del es tinha anticorpo dirigido contra os linfócitos B do doador quenão puderam ser detectados pela técnica CDC tradicionalmente empregada. (Figura 1)

Foram realizados 26 transplantes combinados (17\%), sendo 22 de 
rim e pâncreas, dois de rim e coração e dois de rim efígado. 0 grupo PCCF + incluiu mais pacientes diabéticos $(65,5 \times 34,5 \% ; P=0,04)$ e transplantes combinados $(22,7 \times 11,0 \% ; \mathrm{P}=0,056)$ do que o grupo PCCF-. Observou-se uma associação entre diabete tipo I e PCCF+ contra linfócitos $B$, com risco relativo de $2,75(1,1-7,0)$.

Os pacientes PCCF - e PCCF + foram semel hantes quanto às características demográficas, tipo de transplante e de imunossupressão utilizada, risco imunológico pré-transplante e evolução clínica após a realização do transplante. Estes resultados

Figura 1. Descrição da População Avaliada Retrospectivamente Através de Prova Cruzada por Citometria de Fluxo (PCCF)

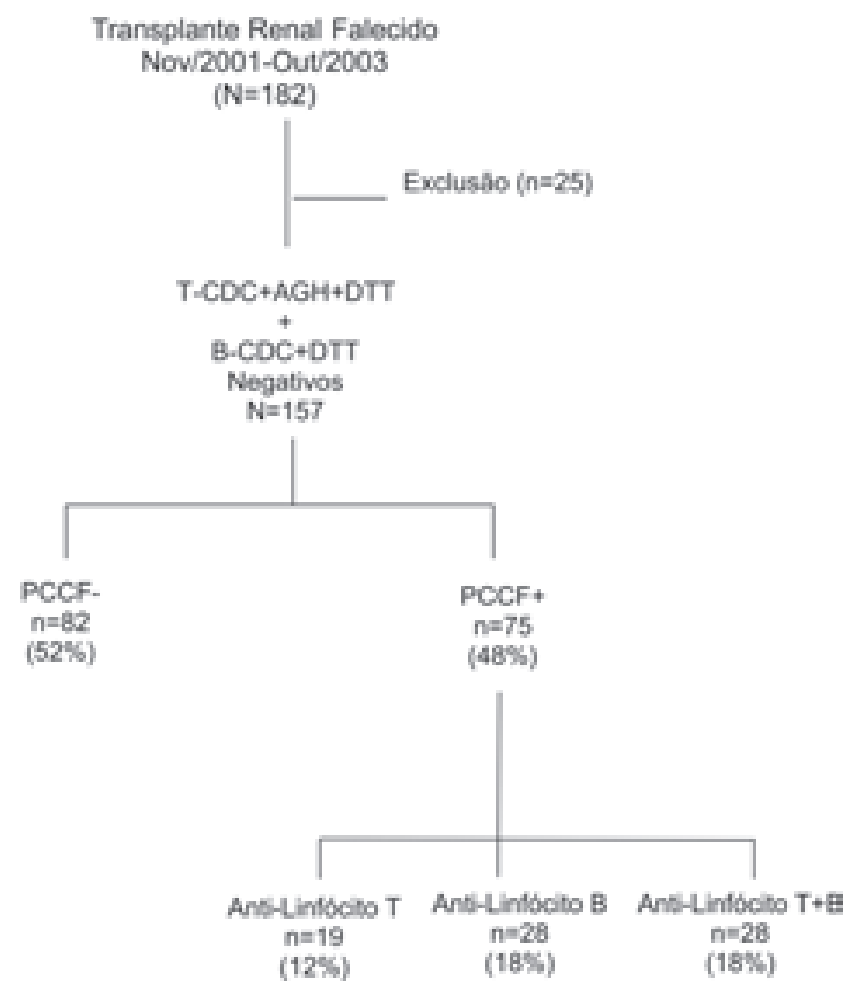

estão descritos naTabela 1.0 protocolo inicial imunossupressor não foi diferente entre os grupos, sendo a associação mais comumente empregada ciclosporina, mofetil micofenolato e prednisona $(52,4 \%$ nos pacientes $C F-$ e $42,7 \%$ nos pacientes $C F+$ ).

A incidência de ENF entre pacientes com PCCF + foi duas vezes mais alta do que em pacientes com PCCF-, mas isso não foi estatisticamente significativo $(12,0 \% \times 6,1 \% \mathrm{P}=0,264)$. A s causas para EN F puderam ser identificadas em seis pacientes com PCCF + (uma trombose vascular, uma rejeição aguda grave com síndrome hemolítico-urêmica e quatro mortes ainda sob tratamento dialítico) e em quatro pacientes com PCCF- (uma trombose vascular, uma rejeição aguda com necrose cortical e duas mortes no primeiro mês ainda sem função do enxerto). Dentre os oito pacientes hipersensibilizados (PR A $>75 \%$ ), cinco tiveram PCCF + $(62,5 \%$ ) e dois deles (40\%) nunca puderam manter-se sem diálise. Dentre os 24 pacientes PRA +, nove $(37,5 \%)$ apresentaram PCCF + no momento do transplante renal e quatro $(44,4 \%)$ deles evoluíram como ENF. Fatores de risco imunológicos e desfechos clínicos, de acordo com a positividade da PCCF, contra linfócito T e/ou B, podem ser observados na Tabela 2.
Tabela 1. Características demográficas e clínicas e evolução do transplante renal conforme o resultado da prova cruzada pré-transplante por citometria de fluxo

\begin{tabular}{llll}
\hline & $\begin{array}{l}\text { PCCF+ } \\
(\mathrm{n}=75)\end{array}$ & $\begin{array}{l}\text { PCCF- } \\
(\mathrm{n}=82)\end{array}$ & $\mathrm{P}$ \\
\hline Gênero Masculino (n; \%) & $44(58,7)$ & $46(56,0)$ & 0,750 \\
Média de idade (anos, média+DP) & $45,5+13,8$ & $43,0+12,0$ & 0,224 \\
Gestação Prévia* (n; \%) & $12 / 25(48,0)$ & $13 / 30(43,3)$ & 0,790 \\
Transfusão de sangue prévia** & & & \\
(n; \%) & $42 / 73(57,5)$ & $44 / 79(57,5)$ & 0,871 \\
PRA+ (n=24) (n; \%) & $9(12,0)$ & $15(18,3)$ & 0,375 \\
PRA>75\% (n=8) (n; \%) & $5(6,7)$ & $3(3,6)$ & 0,480 \\
Re-transplante (n; \%) & $4(5,3)$ & $4(4,9)$ & 1,000 \\
>3 HLA-MM(n; \%) & $37(49,3)$ & $32(39,0)$ & 0,259 \\
Tempo de isquemia fria & & & \\
(h; meas+SD) & $20,1+6,4$ & $20,6+5,1$ & 0,541 \\
Terapia de Indução (n; \%) & $43(57,3)$ & $47(57,3)$ & 1,000 \\
Doador Falecido Limítrofe (n; \%) & $23(30,7)$ & $32(39,0)$ & 0,316 \\
ENF (n; \%) & $9(12,0)$ & $5(6,1)$ & 0,264 \\
FRE (n; \%) & $49(65,3)$ & $58(70,7)$ & 0,497 \\
RAC (n; \%) & $30(40,0)$ & $38(46,3)$ & 0,519 \\
Perda do enxerto no primeiro ano & & & \\
(n; \%) & $13(17,3)$ & $12(14,6)$ & 0,668 \\
Óbito no primeiro ano (n; \%) & $8(10,7)$ & $8(9,8)$ & 1,000 \\
\hline
\end{tabular}

PCCF: prova cruzada por citometria de fluxo

DP: Desvio padrão

*Variável estudada entre 55 pacientes femininas (12 casos sem informação)

** 5 casos sem informação

PRA: teste de reatividade contra painel

PRA+: PRA positivo por técnica de imunoensaio enzimático

(LAT-M screeening)

PRA $>75 \%$ : PRA quantitativo através de citometria de fluxo (realizado quando PRA+)

HLA-MM: incompatibilidades HLA

ENF: enxerto primariamente nunca funcionante

FRE: função retardada do enxerto

RAC: rejeição aguda celular

A creatinina sérica foi semel hante entre os grupos PCCF- ePCCF + nos diferentes tempos analisados durante o primeiro ano póstransplante. 0 nível mais baixo de creatinina sérica atingida no período de estudo também não diferiu entre os grupos. Da mesma forma, PR A + isolado (independente dos resultados da PCCF) não afetou a função do enxerto no primeiro ano. A sobrevida do enxerto no primeiro ano foi semel hante entre os grupos estudados e isso está demonstrado na Figura 2. Q uando os transplantes combinados foram excluídos da análise, os mesmos resultados foram obtidos.

\section{Análise de subgrupos de pacientes \\ - PRA Positivo (Screening LAT-M)}

Pacientes com PR A + na avaliação pré-transplante constituíram $15,3 \%(n=24)$ da população estudada, e $37,5 \%(n=9)$ deles foram submetidos a transplante renal com PCCF +, comparados a 49,6\% dos pacientes com PR A - $(P=0,375)$. Os resultados das PCCF nestes 